\title{
Commentaries
}

\section{Outcome measures for glaucoma treatment}

This article looks at outcome measures for the glaucomas and makes suggestions on how they may be used to guide long term management.

Glaucoma is the term given to diseases of the optic nerve head which exhibit a characteristic deformity of the optic disc (glaucomatous cupping) with loss of retinal neurons together with commensurate visual loss. The intraocular pressure (IOP) may be above the upper limit of normal.

The relation between elevated IOP and glaucomatous cupping is worryingly uncertain. Total population studies suggest an increasing incidence with increasing IOP, but the rise for each unit of IOP increase only becomes dramatic above $30 \mathrm{~mm} \mathrm{Hg.}{ }^{1}$ Additionally, a significant proportion of patients with chronic glaucoma will have an IOP within the normal range. ${ }^{12}$ The susceptibility of an eye to a particular IOP level clearly varies, patient A being more susceptible to a particular IOP level than patient B. This susceptibility may well vary with time; other, perhaps circulatory, factors will also play a part. Together they constitute risk factors of varying significance for the development of glaucoma.

Care for the glaucomas should be directed towards removing the causative factor. Rarely (for example, steroid induced glaucoma) is this possible. More usually no treatable factor is to hand, and treatment therefore is directed towards removing a significant risk factor (elevated IOP). Successful treatment has been seen as achieving a 'target pressure'. ${ }^{34}$ A considerable body of literature exists on the merits of different treatments in achieving and maintaining a particular IOP level. However, as IOP can only be considered a risk factor, we ought to look for the outcome of this or any other treatment upon the rate of change in either visual function or, better still, upon the optic nerve.

In the absence of a natural history of glaucoma, there is no control group against which to measure treatment. As a natural history study may never materialise, the only sensible alternative is to use each patient as his or her own control. In this way the effect of treatment can be measured against the known rate of progression in the untreated or previously treated condition. Fortunately both visual loss ${ }^{5}$ and neuroretinal rim area change ${ }^{6}$ appear to be linear, allowing estimates of progression in an individual patient, together with predictions of time to significant change (for example, loss of a driving licence).

As a result of the above discussion it is possible to identify non-IOP related outcome measures.

This has to be a 'within eye' change in observed disease progression. Theoretical outcome measures related to treatment are threefold.

(1) Optic disc-identify a reduction in the rate of enlargement of the optic cup/reduction in neuroretinal rim area/reduction in rate of decline (thinning) in the thickness of the retinal nerve fibre layer. This has been achieved using planimetric measurements of the area of the neuroretinal rim, although it is labour intensive and open to observer error. Both the scanning laser ophthalmoscope, the nerve fibre layer analyser, and the glaucomascope may be shown to provide this information in the future.

(2) Visual function - white on white perimetry will identify a change (reduction) in the rate of decline in retinal sensitivity at individual retinal locations. This is both practical and simple using pointwise linear progression (Progressor) ${ }^{7}$ and can be based on change in mean slopes of significantly progressing locations. Blue on yellow perimetry may become a practical way of identifying development of reduced visual function before it is visible using white on white perimetry. These outcome measures can be used to show that treatment is having a beneficial effect on the course of the disease.

(3) Side effects - unwanted treatment effects need to be remembered. These may be the effect on the eye (such as visual loss from cataract after glaucoma surgery), ${ }^{8}$ or on the individual (such as reduced exercise tolerance with $\beta$ blocker therapy). ${ }^{9}$ The elderly patient with slowly progressing disease may be worse off with treatment than without. Incremental treatment bringing appreciable morbidity could only be justified if the rate of visual decline without it could affect the patient's quality of life in the expected lifespan.

Outcome measures should be the yardstick by which glaucoma treatment is judged. When lowering IOP in the patient with chronic glaucoma we should set a target pressure, and then use an outcome measure to decide whether it is correct for that patient.

R A HITCHINGS

Moorfields Eye Hospital, London

1 Sommer A, Tielsch JM, Katz J, Quigley HA, Gottsch JD, Javitt J, et al. Relationship between intraocular pressure and primary open angle glaucoma among white and black Americans. The Baltimore Eye Survey. Arch Ophthalmol 1991;109:1090-5.

2 Dielemans I, Vingerling JR, Wolfs RCW, Hofman A, Grobbes DE. The prevalance of primary open angle glaucoma in a population based study in

3 Palmberg P. Epidemiology of POAG and rationale for therapy. Glaucoma Abstracts 1989;6:10-23.

4 Anstracts 1989;6:10-23. Anderson DR. Glaucoma: the damage caused by pressure. XI

5 McNaught AI, Crabbe DP, Fitzke F, Hitchings RA. Modelling sensitivity McNaught AI, Crabbe DP, Fitzke F, Hitchings RA. Modelling sensitivit
loss in low tension glaucoma. Invest Ophthalmol Vis Sci 1994;35:2183.

6 Airaksinen PJ, Tuulonen A, Alanko HI. Rate and pattern of neuroretinal rim area decrease in ocular hypertension and glaucoma. Arch Ophthalmol 1992; 110:206-10.

7 Fitzke F, Hitchings RA, Poinoosawmy D, McNaught A, Crabbe DP. Analysis of visual field progression in glaucoma. Br f Ophthalmol 1996;80:40-8.

8 Schneider P, Flammer J, Hendrickson P. Does glaucoma surgery accelerate cataract development? Glaucoma 1994;16:6-11.

9 Diggory P, Cassels Brown A, Vail A, Abbey LM, Hillman JS. Avoiding unsuspected respiratory side-effects of topical timolol with cardioselective or sympathomimetic agents. Lancet 1995;345:1604-6. 